\title{
Corrigendum: Genome-wide association study identifies new susceptibility loci for epithelial ovarian cancer in Han Chinese women
}

Kexin Chen, Hongxia Ma, Lian Li, Rongyu Zang, Cheng Wang, Fengju Song, Tingyan Shi, Dianke Yu, Ming Yang, Wenqiong Xue, Juncheng Dai, Shuang Li, Hong Zheng, Chen Wu, Ying Zhang, Xiaohua Wu, Dake Li, Fengxia Xue, Haixin Li, Zhi Jiang, Jibin Liu, Yuexin Liu, Pei Li, Wen Tan, Jing Han, Jiang Jie, Quan Hao, Zhibin Hu, Dongxin Lin, Ding Ma, Weihua Jia, Hongbing Shen \& Qingyi Wei

Nature Communications 5:4682 doi: 10.1038/ncomms5682 (2014); Published 19 Aug 2014; Updated 17 Dec 2014

The original version of the Supplementary Information attached to this Article contained an error in the numbering of the Supplementary Tables. The HTML has now been updated to include a corrected version of the Supplementary Information. 